\title{
La administración romana ante la gestión de residuos y tutela del hábitat
}

JosÉ LUIS ZAMORA-MANZANO1

\section{RESUMEN}

Desde un punto de vista medioambiental, los problemas que normalmente se producen en las grandes ciudades están relacionados con los residuos, dada la concentración de viviendas y locales en un espacio reducido. Roma no supone una excepción, de ahí que haya existido una preocupación por la higiene y la gestión y evacuación de residuos urbanos, y que aún hoy en día siguen generando numerosos problemas. En el presente trabajo se hace una aproximación a algunas vicisitudes relacionadas con la gestión de residuos.

Palabras clave: Medio ambiente, Contaminación, Administración romana, Residuos urbanos, Disposición de residuos, Reciclaje.

\section{Roman Administration for Waste Management and Habitat Protection}

\section{ABSTRACT}

From an environmental perspective, problems usually arising in large cities are often related to waste, due to a large group of residencies and business es-

1 Doctor en Derecho y profesor titular de Derecho Romano en la Universidad de las Palmas de Gran Canaria, Las Palmas de Gran Canaria, España. Profesor tutor del Centro Asociado de las Palmas de la Universidad Nacional de Educación a Distancia de Madrid, Las Palmas de Gran Canaria, España. Correo-e: joseluis.zamora@ulpgc.es Fecha de recepción: 2 de julio de 2016. Fecha de modificación: 27 de agosto de 2016. Fecha de aceptación: 15 de septiembre de 2016. Para citar el artículo: Zamora-Manzano, J. L. "La Administración romana ante la gestión de residuos y tutela del hábitat", Revista digital de Derecho Administrativo, n. ${ }^{\circ} 17$, primer semestre, Universidad Externado de Colombia, 2017, pp. 69-87. DOI: http://dx.doi.org/10.18601/21452946.n17.06. 
tablishments in a small space. Rome is no exception, hence it has historically been concerned about hygiene and the management and disposal of urban waste, which continues to present day, generating numerous problems. This paper will address some of the vicissitudes related with waste management.

Keywords: Environment, Pollution, Roman Administration, Municipal Waste, Waste Disposal, Recycling.

\section{INTRODUCCIÓN}

En las líneas que siguen pretendemos desarrollar algunas consideraciones sobre la gestión de residuos y otros aspectos relacionados directamente con la problemática del hábitat de las ciudades. Trataremos de enhebrar una argumentación lógica del estudio de las diferentes fuentes para constatar, por medio de un estudio interdisciplinar, que la problemática que sufrimos hoy en día en la gestión de residuos y lixiviados era ya un problema que afectaba a la Administración romana. En este sentido, como indica mi maestro, el profesor Don Antonio Fernández de Buján, catedrático de Derecho Romano de la Universidad Autónoma de Madrid, con la auctoritas que le confiere ser el máximo exponente en España de las líneas de investigación relacionadas con el derecho administrativo y fiscal romano y director de una de las colecciones más importantes sobre monografías de derecho romano y culturas clásicas de la editorial Dykinson, es necesaria la reconstrucción del derecho administrativo romano, con lo que ello comporta: la conexión entre investigación histórica y dogmática moderna².

Los problemas derivados de las agrupaciones humanas en las ciudades siempre han originado residuos dada la concentración de viviendas ${ }^{3}$ y locales en un espacio reducido ${ }^{4}$. Roma no constituye una excepción, de ahí que haya

2 Antonio Fernández de Buján, Derecho público romano, Madrid: Civitas, 15. a ed., 2016, pp. 239 ss.; vid. también Id., "Instituciones, hechos y actividad de orden administrativo en la experiencia jurídica romana", Xornadas e Seminarios: Derecho Administrativo Histórico, 1, 2005, pp. 19-158.

3 Vitrubio, De arch 1.1.10: Disciplinam vero medicinae novisse oportet propter inclinationem caeli, quae Graeci climata dicunt, et aeris et locorum, qui sunt salubres aut pestilentes, aquarumque usus, sine bis enim rationibus nulla salubris babitatio fieri potest. Vitruvio en su tratado de arquitectura ya habla de la importancia del lugar y del clima, aludiendo a las características del aire, lugar, aguas, salubridad. Vid. en este sentido SASCHA PRIESTER, Ad summas tegulas, Unterchungen zu vielgeschossigen Gebäudeblöcken mit Wobneinbeiten und Insulae im Kaiserzeitlichen Rom, Roma, 2002.

4 Alrededor del siglo II a. C. la población rural empieza a emigrar y a concentrarse en los grandes núcleos urbanos, motivada, en muchos casos, por la crisis de los pequeños y grandes propietarios ante los latifundios, la competencia de los productos provinciales de coste inferior y el escaso mercado artesanal que no lograba atraer a este proletariado. Vid. PaOla PISAnI. Uomo, natura, ambiente nella letteratura latina, Genova: Compagnia dei librai, 1990, p. 28. 
existido una preocupación por la higiene y la gestión y evacuación de residuos urbanos, temática que hoy en día sigue siendo motivo de desasosiego por los problemas medioambientales que genera.

La sociedad urbana romana tampoco se libró de este problema, y de hecho en una de sus colinas, el monte Testaccio o monte dei $\operatorname{cocci}^{5}$, se encontraba un inmenso vertedero de envases de alimentos, vino y aceite, compuesto en su gran mayoría por restos cerámicos, normalmente ánforas. En este contexto, conscientes del problema e impacto medioambiental que se producía, vamos a abordar de qué manera afrontó el derecho romano estas vicisitudes, sabedores de la complejidad y de la falta de homogeneidad en las fuentes, conociendo que existe todo un conjunto de normas en el ordenamiento jurídico romano, con muchas referencias directas e indirectas pero muy dispersas, que aluden al problema del hábitat ${ }^{6}$ y el medio ambiente ${ }^{7}$.

Desde época remota existe una preocupación por la salubritas ${ }^{8}$ y el hábitat de las urbes, así como por todo lo relacionado con el agua: los desagües, la circulación, la asistencia y servicios públicos, entre otros; en una palabra, todo lo que se vincula con el funcionamiento de una comunidad organizada. Así, en la ciudad de Roma encontramos un grave problema de enjundia, como es la congestión en las vías públicas y también el hacinamiento en las insulae, desde las cuales, muchas veces, se arrojaban basuras, como veremos más adelante, fenómeno que implicó una intervención normativa, junto a otras medidas, a fin de poner solución a todos aquellos inconvenientes que, de forma exponencial, se iban produciendo con el desarrollo y la creciente concentración urbana.

En el derecho romano no existió una tutela directa del medio ambiente, pero sí indirecta por medio de una regulación específica que disciplinó la salubridad de las aguas, minas e incluso la deforestación ${ }^{9}$, y, como veremos acto seguido, la gestión de los residuos.

5 La colina alcanzó unos 30 metros de altura y se formó por acumulación de los restos de ánforas que eran llevados allí, dando origen a un auténtico vertedero. Este, como sostiene Filippo Coarelli, Roma, Roma-Bari: Laterza, 1981, p. 340, "costituiscel'archivio, ancora in gran parte inesplorato, della storia economica de Roma". Las ánforas llegaban del puerto y se depositaban y rompían allí con restos de recipientes que procedían de aceites y vinos, por ello normalmente se enterraban en cal para evitar los malos olores. Sobre las ánforas son dignos de mención los estudios de HeINRICH Dressel, Inscriptiones Urbis Romanae Latinae-Instrumentum domesticum 1-2, Berlin, 1966-1975.

6 Las patologías de las que adolece el urbanismo romano se deben a la enorme presión de mográfica que hace que se desarrollen suburbios con calzadas estrechas para el tránsito, lo que constituyó un foco de propagación de incendios, principal motivo para la reordenación de la ciudad.

7 Laura Solidoro Maruotti, La tutela dell'ambiente nella sua evoluzione storica, L'esperienza del mondo antico, Torino: Giappichelli, 2009, p. 41.

8 Emilio Albertario, "L'uso traslato di salubris, salubritas, salubriter nelle fonti giuridiche romane", en Studi di Diritto romano, vol. 6, pp. 183 ss.

9 RenATE FisCHER. Umweltschützende Bestimmungen im Römischen Recbt, Aachen: Shaker, 1996, pp. 3 ss 
Centrándonos en nuestro análisis sobre el problema de la gestión de residuos urbanos, antes de acometer la problemática de su gestión, debemos formular una definición de lo que se entiende por dicha actividad ${ }_{i}$ así entendemos por la misma: "aquella en la cual, hoy en día, se recolecta, transporta, procesa y recicla todo material orgánico o no de desecho generalmente producido por la actividad humana, en un esfuerzo por reducir los efectos perjudiciales en la salud humana y la estética del entorno".

Hoy en día contamos con la Ley 22/2011, de 28 de julio, de residuos y suelos contaminados, cuyo objeto es regular la gestión de los residuos impulsando medidas que prevengan su generación y mitiguen los impactos adversos sobre la salud humana y el medio ambiente asociados a su generación y gestión, mejorando la eficiencia en el uso de los recursos; la ley define residuo (art. 3) como cualquier sustancia u objeto que su poseedor deseche o tenga la intención o la obligación de desechar. Veamos a continuación una breve aproximación a la gestión y su problemática en relación a la tutela del hábitat en el derecho romano.

\section{UNA BREVE APROXIMACIÓN A LA GESTIÓN DE RESIDUOS Y LA VIGILANCIA DEL ESPACIO URBANO}

Existen algunas evidencias indirectas sobre la manera como se organizaba la gestión de residuos ${ }^{10}$ y la problemática que esta ocasionaba al medio; así encontramos el senadoconsulto de Pago Montano en el cual ya se hace referencia a evitar el amontonamiento de desperdicios y basuras:

eisque curarent tu[ erenturque/ar] bitratu aedilium pleibeium, [quei] comque essent, neiue ustrinae ${ }^{11}$ in leis loceis regionibusue niue poci ustri nae cussa fierent, niee stercus terra $[\mathrm{m}]$ ue intra ea loca fecisse

10 La gestión de residuos urbanos y su problemática en las ciudades griegas es puesta de relieve

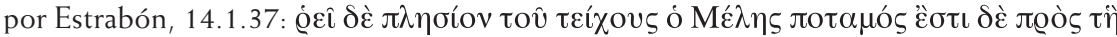

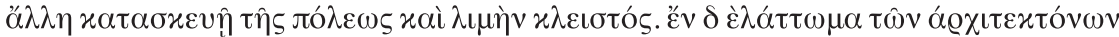

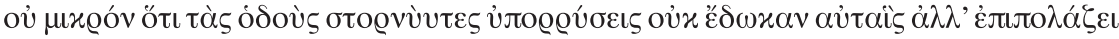

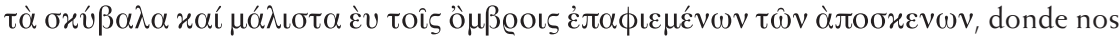
informa que la falta de previsión de un sistema de drenaje y alcantarillado subterráneo hizo que los residuos y basuras se acumularan en las calles. Sin embargo nos encontramos con previsiones como la ley de Pérgamo, que obliga a los habitantes a limpiar y reparar las vías aledañas; para el estudio y comentario de la ley, vid. Ana Rodrícuez GonZález. "Reception of Law: derecho y espacio urbano en dos ciudades de la antigüedad", Rida, 2012, 59, pp. 23 ss.

11 La disposición también contempla de forma inicial el alejamiento de los lugares de incineración de cadáveres, una medida que contribuyó a alejar el fantasma de los incendios desde época arcaica, vid. CiCERón, De Leg. 2.23.58, Credo vel propter ignis periculum. Quod autem addit 'neve urito', indicat non qui uratur sepelin, sed qui bumetur. Credo vel propter ignis periculum. 
coniecisseue veli $[t]$ quei baec loca ab paa [g]o Montano//[reddempta babebit, et uti si is stercus in eis loceis fecerit terramue in ea] loca iecerit, in ... [cum HS] ma]nus iniectio pignorisq(ue) capi[ o siet $]^{12}$.

En Roma, el Pago Montano era un lugar de enterramiento ${ }^{13}$ de los plebeyos más allá de la Puerta Esquilina, y lo que se trató de impedir fue la formación de un vertedero por acumulación de residuos. El senadoconsulto, de fecha incierta, acaso del siglo I, comenta dos de los procedimientos ejecutivos que se pudieron aplicar a los infractores para el pago de una multa ${ }^{14}$. En este mismo contexto, en el año 87 a. C., encontramos el edicto praetoris de Campo Esquilino ${ }^{15}$ :

L. Sentius C. f. pr(aetor)/ de sen (atus) sent(entia) loca / terminanda coer(avit) B(onum)f(actum). Nei quis intra/ términos propius/urben ustrinam/fecisse velit neiue/ stercus cadauer/iniecisse velit

Existen fragmentos en las fuentes literarias que ya mencionan la evacuación de residuos fuera de los confines de la ciudad, como nos informa Tácito, Ann. 15.43. $1^{[16]}$; así, las barcas que se encargaban de transportar el frumentum aprovechaban el viaje de vuelta para ser cargardas con escombros y residuos que eran depositados en las marismas cercanas a Ostia.

A mayor abundamiento, también existen referencias a los carros que desempeñaban el servicio de limpieza y gestión de residuos en Tácito, Ann. 11.32 ${ }^{[17]}$, al referirse a la fuga de Mesalina Luculianos que logra escaparse en un carro de los destinados a la limpieza (vebiculo, quo purgamenta bortorum).

13 Con posterioridad existen numerosas intervenciones legislativas por parte de los emperadores como Adriano (Ulpiano 25 ad. Ed., D. 47.12.3.5), que establece una pena de 40 áureos para los que entierran en la ciudad, y la misma para los magistrados que lo permitieren, ordenando además que el lugar sea confiscado y los cadáveres trasladados: Divus badrianus rescripto poenam statuit quadraginta aureorum in eos qui in civitate sepeliunt, quam fisco inferri iussit, et in magistratus eadem qui passi sunt, et locum publicari iussit et corpus transferri. Con posterioridad se prohibió, a fin de preservar las condiciones de salubridad, guardar cadáveres dentro de la ciudad, estableciéndose incluso el traslado de los féretros según establece en una providencia de los emperadores, sancionándose con 50 libras de oro; vid. C.Th. 9.17.6.

14 Vid., sobre el carácter popular del SC, Giovanni Pugliese. "Figure processuali ai confini tra iudicia privata e iudicia publica", Studi Solazzi, Napoli, 1948, p. 411, quien sostiene que "per $i$ Romani il proceso per multa era un iudicium publicum, ma nessuna fonte si esprime in questo modo esplicito, solo Cicerone nel in Verr. 2.1.60, 155". En igual sentido, vid. Franco Casavola. Studi sulle azioni popolari romane. Le actiones populares, Napoli, 1958, pp. 69 ss.

15 Fira I, 53, p. 306.

16 ... eas proticus Nero sua pecunia exstructurum purgatasque areas dominis traditurum pollicitus est. addidit praemia pro cuiusque ordine et rei familiaris copiis, finivitque tempus, intra quod effectis domibus aut insulis apiscerentur. ruderi accipiendo Ostienses paludes destinabat, utique naves, quae frumentum Tiberi subvecta[v] issent, onustae rudere decurreren. .

17 ... id repente solltudinis erat-spatium urbis pedibus emensa, vehiculo, quo purgamenta hortorum eripiuntur, Ostiensem viam intrat nulla cuiusquam misericordia quia flagitiorum deformitas praevalebat. 
Ahora bien, sin perjuicio de otros fragmentos que analizamos a continuación, en Roma existió también el aprovechamiento de residuos sólidos por medio del reciclaje de cerámica y cristal; así existían las lagunariae ${ }^{18}$ encargadas de vender y comercializar las lagenae, unas ánforas de diseño circular y cuello estrecho destinadas normalmente a contener vino.

También en relación con la reutilización de envases cerámicos, otros eran empleados como productos de revestimiento, como opera signina ${ }^{19}$ o cimentación hidráulica para encofrado de cisternas, lacus, piscinae y otros elementos arquitectónicos y edificios que debían soportar la humedad; por tanto, se realizaba con todas las ánforas, principalmente, un amasijo o conglomerado ${ }^{20}$ que se destinaba a material de construcción.

Volviendo a la gestión de residuos, Papiniano, respecto de las competencias de los ediles, en D. 43.10.1.5, comenta que los ediles debían velar

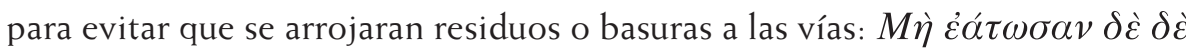

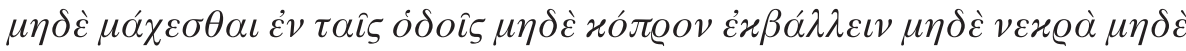
$\delta \varepsilon ́ \varrho \mu \alpha \tau \alpha \varrho \iota \pi \tau \varepsilon \iota \nu$; la previsión edictal que nos comenta el jurista va dirigida a

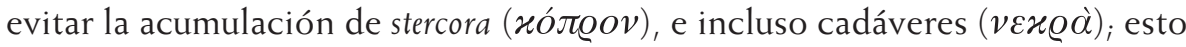
último probablemente referido a cuerpos de animales y sus pieles, que podían causar hedor por su estado de descomposición y putrefacción al quedar hacinados en las calles. Por ello, es primordial gestionar los residuos adecuadamente, ya que estos pueden afectar al tránsito de las ciudades, y al propio ambiente, sobre todo por contaminación atmosférica, pudiéndose generar malos olores por descomposición de los restos orgánicos.

Asimismo, sabemos que cualquier actividad antropogénica, por lógica, conlleva la generación de residuos sólidos, y el problema que afecta también a la Administración romana es que la mayoría no son reincorporados a los procesos productivos, salvo excepciones, provocando en los vertederos y lugares

18 En relación a las ánforas, que constituyen uno de los contenedores comerciales estándar de la época, debemos señalar la existencia de dos clases: las multiuso, destinadas a fruta y vino, y otras de un solo uso, para aceite y garum. Las primeras, normalmente, eran reutilizables, y existen fuentes que atestiguan el uso y reciclaje de estos contenedores; así existían las lagonarii o lagunarius, que se encargaban de esta función entre el Aventino y el Tíber, por tanto cerca del Foro Boario, en las murallas Servianas, como se desprende de CIL VI. 9480 LeOnTIA QVe DefVCta est IDVS SePtbene merenti in PaCe ad Porta Trigemina Lagunara.

19 ElIO de MAGISTRIS. Structurae, ricerche su tecniche costruttive e monumenti antichi, Napoli: Loffredo, 2010, pp. 111 ss.

20 Sobre este conglomerado se pueden citar dos fuentes de referencia: Vitrubio, De arch. 8, 6, 14: sin autem loca dura erunt aut nimium venae penitus fuerint, tunc signinis operibus ex tectis aut superioribus locis excipiendae sunt copiae. in signinis operibus baec sunt $f a-c i e n d a$. uti harena primum purissima asperrimaque paretur, caementum de silice frangatur ne gra- vius quam librarium, calce quam vebementissima mortario mixta ita ut quinque partes barenae ad duas respondeant. eo tum fossa ad libramentum altitudinis quod est futurum calcetur vectibus ligneis ferratis. parietibus calcatis in medio quod erit terrenum exinaniatur ad libramentum infi-mum parietum. boc exaequato solum calcetur ad crassitudinem quae constituta fuerit. En igual sentido, vid. PLINIO, Nat. bist, 36, 173. 
donde se acumula basura -fundamentalmente restos orgánicos- la aparición de lixiviados, líquidos que se forman como resultado de pasar o percolarse a través de un sólido, arrastrando distintas partículas potencialmente contaminantes.

A mayor abundamiento, es importante exponer en materia de gestión de residuos y mantenimiento de las vías los pasajes de la polémica ${ }^{21}$, y no exenta de interrogantes ${ }^{22}$, Tabulae Heracleensis ${ }^{23}$ :

1. 20-23 Quae viae in rubem Rom(am) propiusue u(rbem) $R$ (omam) p(assus) Mubei continente babitatibur sunt erunt, quoius ante aedificium earum quae viae erunt, is eam niam arbitratu eius aed(ilis), quoi ea pars urbis b(ac) l(ege) obuenerit, tueatur, isque aed (dilis) curato uti quorum ante aedificium erit quamque niam $b(\mathrm{ac}) \mathrm{l}$ (ege) quemque tueri oportebit, ei omnes eam uiam arbitratu eius tueantur, neue eo loco $a<q>$ ua consistat, quo minus conmode populus ea uia utatur.

Si observamos el fragmento de la tabula, a priori, la disposición establece la tutela y vigilancia por parte de los ediles ${ }^{24}$; la fuente habla del mantenimiento de las vías en Roma pero no de su limpieza, la purgatio ${ }^{25}$. Además de la tutela y control de la urbe y periferia y núcleo suburbano dentro de los mil pasos

21 Sobre la Tabla de Heraclea, descubierta en 1732, existe abundante literatura que se detiene en los problemas que presenta esta ley, respecto de su aplicación general o de ámbito municipal, ya que muchas normas hacen referencia a la ciudad de Roma; respecto de los problemas en cuanto a su armonización de contenidos per saturam rogare; e incluso en lo que hace a su datación, donde, en opinión de Álvaro d'Ors, "Nuevos datos de la ley Irnitana sobre jurisdicción municipal", SDHI, 49, 1983, pp. 20-21, "tenemos un dato seguro para afirmar que Augusto dio una ley municipal, y nada impide pensar que se trata de la lex Iulia Municipalis que suele atribuirse a César. La verdad es que esta atribución se había establecido en base a una identificación de esa ley con la Tabula Heracleensis, pero, una vez que esta identificación parece equivocada, no hay inconveniente en atribuir esa ley a Augusto y no a César". En este sentido, el autor se apoya en la inscripción CIL v 2864 = Dessau 5406, que se puede referir más, por la época, a Augusto. Excedería de nuestro cometido hacer un análisis sobre todos los problemas que suscita la misma.

22 Se plantea incluso si realmente el documento tenía una sanctio y si del final de la disposición (1. 159 ss.) se infiere que se trata de un pseudo estatuto: "it as a particular sort of quasi-legal document, with for example a quasi sanctio; and thought analysis of the implications inberent both in wabt it is, and what it pretends to be", según EDward Bisphant. "The End of the Tabula Heracleensis a por man's sanctio?", Epigrapbica, 59, 1997, p. 156. Aunque existen tesis dispares sobre si fueron normas romanas elaboradas por los propios heracleotas con fines prácticos o si fue, por ejemplo, una ley de confirmación general de legislación cesariana; lo cierto es que hasta su naturaleza jurídica plantea numerosos interrogantes sobre si además se trató de una ley que regularía la jurisdicción local en los municipios de toda Italia.

23 La edición que manejamos es la de Michael H. CrawFord. Roman Statutes, vol I, London, 1996, pp. 354 ss.; sobre literatura p. 355. Id., Fira I. 240, CIL $1.206=593$.

24 Además, a los cinco días de su nombramiento deben sortear la parte de la urbe hasta los mil pasos que le corresponden a cada uno para el ejercicio de su competencia. 1. 24 Tab. Her.: iei in diebus $V$ proxumeis quibus eo mag (istratu) designatei erunt eumue mag(istratum) inierint inter se paranto aut sortiunto, quae in partei urbis quisque eorum uias publicas in urbem Roma(m) p(assus) $M$ reficiundas sternendas curet, eiusque reiprocurationem babeat.

25 Silvio Panciera. "Nettezza urbana a Roma. Organizzazione e responsabile", en Sordes Ur- 
próximos a esta, la disposición trata de garantizar también un tráfico fluido en la vía al señalar neue eo loco $a<q>$ ua consistat, quo minus conmode populus ea uia utatur. La ley obliga a los propietarios colindantes con la vía a mantener en buen estado la misma; la expresión que utiliza el texto es "tueri" (tueor-eri, tuitus), mantenimiento bajo la observancia de los ediles con competencia en la zona correspondiente.

Existe, por tanto, una prohibición de alterar la vía pública, con una obligación de hacer la refectio o reparación, bajo la vigilancia del edil, ya que en caso de no hacerlo se procedía a la adjudicación a terceros. De esta forma, la ley ${ }^{26}$ dispone que el edil debe, diez días antes de arrendar la obra de reparación, anunciar en el foro cuál es la vía afectada, y muchas veces podía darse el caso de que esta fuese impracticable por tener agua estancada, neиe eо loco $a<q>$ ua consistat, en el día en que se haya de arrendar a esos terceros, con notificación a los propietarios afectados bien en sus domicilios o a través de sus procuradores, erit procuratoribusue eorum domun denuntietur facito. En dicho procedimiento, según se infiere de la norma, era necesaria la intervención de cuestor u otro magistrado a cargo del Erario. Ahora bien, una de las singularidades que se destacan en el procedimiento ${ }^{27}$ es la necesidad de llevar libro de registro en el que conste el valor de la obra arrendada, estableciéndose cómo imputar los costes a los propietarios en función de la longitud de la vía ocupada por la fachada de los edificios colindantes y la anchura de la calle; de esta forma se procedía, por medio de una adtributio, a vincular al arrendatario contratista con los propietarios de los edificios afectados ${ }_{i}$ la ley daba un plazo de treinta

bis, Roma, 2000, p. 99; el autor señala que "la Tabula non può in alcun modo essere utilizzata per sostenere che era sui cittadini che le autorirità scarivano l'onere di tener pulita Roma".

26 L. 32-45: Quemquomque ante suun aedificium niam publicam $b($ ac) $l($ ege) tueri oportebit, quei eorum eam niam arbitratu aed(ilis), quois oportuerit, non tuebitur, eam niam aed(ilis), quonius arbitratu eam tuerei oportuerit, tuendam locato; isque aed(ilis) diebus ne minus $X$ antequam locet aput forum ante tribunal suom propositum babeto, quam uiam tuendam et quo die locaturus sit $e<t>$ quorum ante aedificium ea uia sit, eisque quorum ante aedificium ea via erit procuratoribusue eorum domun denuntietur facito, se eam uiam locaturum et quo die locaturus sit, eamque locationem palam in foro per q(aestiorem) urb(anum) eumue quei aerario praerit facito. quanta pecunia eam uiam locauerit, tamtae pecuniae eum eos[q]ue, quorum ante aedificium ea uia erit, pro portioni quantum quoiusque ante aedificium niae in longitudine et in latitudine erit, $q$ (uaestor) urb(anus) queiue aerario praeteri in tabula $<$ s $>$ publicas pecuniae factae referendum $\mathrm{cu}<r>$ ato. $E i<q>\mathcal{u}<e>I$ eam tuendam redemerit, tamtae pecuniae eum eosue adtributio sine $d($ olo $) m(a l o)$. Sei is quei adtributus erit eam pecuniam diebus $X X X$ proxum $<e>$ is, quibus ipse aut procurator eius sciet adtributionem erit, non soluerit neque satis fecerit, is, quamtae pecuniae adtributus erit, tamtam pecuniam et eius dimidium ei, quoi adtributus erit, da $<r>e$ debeto, inque eam $r<e>m$ is, quoquomque de ea re aditum erit, indicem indicimue ita dato, utei de pecunia credita <iudicem $>$ indicium [q] ue dari oporte $<r e>t$.

27 Vid. el amplio análisis de RodríGuez GonZálEZ, ob. cit., p. 397, donde destaca la existencia del procedimiento específico de vinculación de los arrendatarios a los propietarios de los edificios a través de la adtributo y considera (p. 410) que "il convenuto ba pocbe possibilità per evitare la condanna: potrebbe, ad esempio, far valere la solutio del debito, nel caso in cui abbia già effettuato il pagamento, oppure eccepire che il termine di trenta giorni non è scadutto - fatto che determina la perdita della causa da parte dell'attore per pluris petitio". 
días para hacer el pago a los propietarios renuentes contra los cuales se podían dirigir directamente los arrendatarios, para lo cual contaban con iudicium utei de pecunia creditae $\mathrm{y}$, por ende, con la actio creditae pecuniae.

Es evidente que las obras podían estar relacionadas con una mejora en la gestión de residuos urbanos y del transporte del mismo a través de las vías que debían de estar en condiciones óptimas, aunque la expresión que utiliza la tabula habla de reficiundas, sternendas ${ }^{28}$, purgandeis ${ }^{29}$, es decir de mantenimiento de vías ${ }^{30}$, empedrado y limpieza. Además, era necesario también un buen servicio de drenaje y vigilancia de las cloacas que podían desbordarse por falta de limpieza, e incluso el control de la evacuación de residuos a través del lanzamiento ${ }^{31}$ de basuras, que en la mayoría de los casos se solía hacer directamente desde los pisos superiores de las insulae, una práctica incívica sancionada con el edicto de effussis et deiectis.

En relación con estas funciones de observancia la tabla alude, en cuanto a su encomienda, a los ediles ${ }^{32}$ y a los quattuorviri y los duoviri:

1. 50-52: Quo minus aed(iles) et Iluir(ei) uieris in urbem purgandeis, Iluir(ei) uieis extra propiusue urbem Rom(am) passus $<M>$ purgandeis, qeuiquomque erunt, uias publicas purgandas curent eiusque rei potestatem babeant, ita utei legibus pl(ebei)ue sc(itis) s(enatus) c(onsultis) oportet oportebit, $e<$ ius $>b($ ac) $l$ (ege) $n$ (ibilum) r(ogatur).

Del fragmento se infiere que las tareas de supervisión corresponden a ediles, los IVviri y IIviri, con competencias limitadas a lo que establezcan las leyes, los plebiscitos y los senadoconsultos. Por tanto, se establece la competencia de

29 Tab. Her. 1. 50- 51.

30 Antonio Palma. Iura vicinitatis, Solidarietà e limitazioni nel rapporto di vicinato in diritto romano dell'età classica, Torino, 1988, p. 122.

31 Respice nunc alia ac diversa pericula noctis:quod spatium tectis sublimibus unde cerebrum testa ferit, quotiens rimosa et curta fenestris vasa cadant, quanto percussum pondere signent et laedant silicem. possis ignavus baberi et subiti casus inprovidus, ad cenam si intestatus eas: adeo to fata, quot illa nocte patent vigiles te praetereunte fenestrae. Ergo optes votumque feras miserabile tecum, ut sint contentae patulas defundere pelves Juvenal Sat. 3.268-277; en su retrato ácido e irónico de la sociedad romana nos comenta los peligros de la ciudad y sobre todo, en la noche, la necesidad de hacer testamento si se iba a una cena, por los peligros que albergaba el tránsito de la ciudad ante los vertidos que se realizaban desde las insulae. Muchas veces se lanzaban las orinas desde los pisos superiores, ya que en ellos no podían existir latrinae ni pozos negros; solo existía la alternativa de tener lasanae o matellae, recipientes que muchas veces se vertían directamente en los sumideros o cloacas. Debemos tener en cuenta la gran cantidad de residuos urbanos que generaba la ciudad de Roma y que afectaba a la salubridad de toda la ciudad; vid. AlexANDER SCOBIE. "Slums, sanitation and morality in the roman world", Klio, vol. 68, n. ${ }^{\circ} 2,1986$, pp. 412 ss.

32 Vid. sobre las similitudes de la norma con la Tabula Heracleensis (1. 20-79) y la Lex Irnitana (c. 19) en cuanto a las funciones de los ediles, JuLio MANGAS. Leyes coloniales y municipales de la Hispania romana, Madrid, 2001, p. 37. 
estos magistrados que se establecen en los municipios y colonias ${ }^{33}$ ad exemplum urbis y que posteriormente serían sustituidos por los curatores viarum ${ }^{34}$. Si se repara, en el texto no se habla de tuito $^{35}$ sino de purgandeis, a fin de poder controlar las condiciones higiénicas de las calles y mantener la vía en el estado original, cuestión que también se deduce del texto de Ulpiano, en relación con las competencias de los ediles:

68 ad. Ed, D. 43.12.1.1: Viam aperire est ad veterem altitudinem latitudinemque restituere. sed et purgare refectionis portio est: purgare autem proprie dicitur ad libramentum proprium redigere sublato eo quod super eam esset. reficit enim et qui aperit et qui purgat et omnes omnino, qui in pristinum statum reducunt.

El fragmento se ubica dentro del conjunto de medidas interdictales creadas por el pretor con la finalidad de proteger las vías, por ser éstas cosas de dominio público. El texto en cuestión, que se encuentra dentro de la rúbrica sobre el interdicto de via publica et itinere publico reficiendo ${ }^{36}$, contempla lo que implica el reficiere, el mantenimiento de las vías, estableciendo por un lado el aperire viam y el purgare viam, esto es, restablecerla en altura y anchura y por otro lado limpiarla ${ }^{37}$ y quitarle todo lo que hubiese en ella para dejarla tal y como estaba. Es evidente que se podía producir acumulación de residuos sólidos y líquidos, muchas veces debido a la falta de drenaje de las cloacas, quedando las aguas fecales estancadas en la vía; de ahí la importancia de proceder a la vigilancia y control de estas, en las cuales tampoco se podía, como nos comenta el siguiente fragmento, bajo pretexto de mejora para ensanchar la vía o hacerla más alta o más baja, empedrar la que era de tierra y viceversa:

Si quis in specie refectionis deteriorem viam facit, impune vim patietur, propter quod neque latiorem neque longiorem neque altiorem neque bumiliorem viam sub nomine refectionis is qui intercidit potest facere, vel in viam terrenam glaream inicere aut sternere viam lapide quae terrena sit, vel contra lapide stratam terrenam facere ${ }^{38}$.

33 Si observamos la organización municipal de Opitergium, se establecía en relación a nuestra materia que los ediles y los quatuorviri aedilicia potestate ejercitaban la policía de la ciudad y aquella sobre los mercados, auxiliados por los apparitores, verificando los productos en mal estado o la salubridad de los mismos y las vías públicas. GiambattisTa ImPallomeni. "L'inquadramento giuridico delle colonie e dei municipi (Iula Concordia e Opitergium) nell'ambito dell'Impero Romano", Index, 26, 1998, pp. 7 ss.

34 Vid. Warner Eck. Die Verwaltung des Römischen Reiches in der bohen Kaiserzeit, Basel, I, 1995, pp. 290 ss; con abundantes referencias epigráficas.

35 Silvio Panciera. Nettezza urbana, cit., p. 102.

36 Praetor ait: "quo minus illi viam publicam iterve publicum aperire reficere liceat, dum ne ea via idve iter deterius fiat, vim fieri veto".

37 Olivia F. Robinson. Ancient Rome. City planning and administration, London, 1992, pp. 59 ss.; particularmente sobre la limpieza de las calles, vid. pp. 69-73. 
Como ya hemos señalado antes, el texto de Papiniano nos aclara que lo ediles velaban y controlaban el estado de las vías para evitar que se ensuciasen, según se infiere de D. 43.10.1. $5^{[39]}$. Aludiendo, en cuanto al control ${ }^{40}$, a

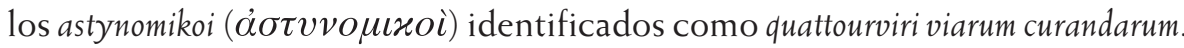

Se aprecia una intervención directa de la autoridad pública, y para ello se instauraron una serie de mecanismos interdictales puestos por la administración ante situaciones en las cuales existía la responsabilidad de los particulares en cuanto a la limpieza y restauración de paredes propias, construcciones particulares que podían afectar a las vías públicas e incluso a la conducción de aguas en los acueductos; de hecho había que dejar expedito el paso y tránsito viario en las obras, o limpiar los acueductos en caso de que estos se vieran afectados por las obras. Ante este tipo de vicisitudes, intervenían los magistrados antes enumerados con la imposición de las correspondientes multas o, como hemos visto, mediante el procedimiento adtributio de ejecución contra los propietarios.

\section{ALGUNAS CONSIDERACIONES SOBRE EL TRANSPORTE Y EL TRATAMIENTO DE LOS RESIDUOS}

Desde nuestro punto de vista, uno de los problemas de gran enjundia a los que tuvo que enfrentarse la Administración fue el transporte de los residuos y basuras que se generaban en la ciudad, a fin de garantizar la salubridad de las vías y acueductos. En este sentido, es evidente que las cloacas ${ }^{41}$ prestaron un servicio de eliminación de residuos donde muchas veces se realizaban vertidos de lasanae o a través de los agujeros a manera de alcantarilla, foramina, que se disponían en la red de cloacas, y además estas estaban tuteladas con un sistema interdictal que trató de preservar también la salud ${ }^{42}$.

A este respecto conviene decir que también en Roma nos encontramos con un servicio de recogida de residuos: por un lado contamos con los foricarii o extractores ${ }^{43}$, que trabajan en la letrinas y también recogían las vasijas

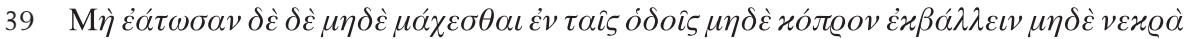
$\mu \eta \delta \dot{\varepsilon} \delta \dot{\varepsilon} \varrho \mu \alpha \tau \alpha \varrho \varrho \iota \tau \tau \varepsilon \iota \nu$

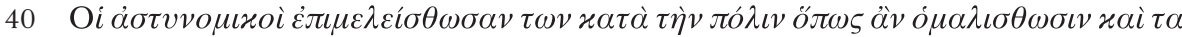

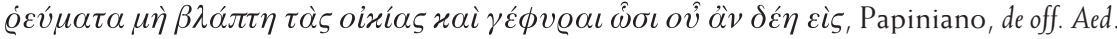
D. $43.10 .1 \mathrm{pr}$.

41 Locus vel fossa extra civitatem in quam publicarum latrinarum stercora egeruntur in quem scilicet locum corpora sanctorum martyrum causa despectionis iactabantur: GOETZ, Corpus Glossarium Latinorum, 1898 , p. 584.

42 Laura Solidoro Maruotti. La tutela dell'ambiente, cit., pp. 87 ss.; "la tutela giurisdizionale si concretizzò in una serie de interdetti de cloacis, intesi ad assicurare il buon funzionamiento delle cloacbe, pubbliche e private".

43 Paulo, lib. sing de Usuris, D. 22.1.17.5, nos comenta los intereses que el fisco cobra de estos, Fiscus ex suis contractibus usuras non dat, sed ipse accipit, ut solet a foricariis, qui tardius pecuniam inferunt, ítem ex vectigalibus cum autem in loco privati successit, etiam dare solet. Sobre la recogida de orina, Marcial, VI. 93.1-2: Tam male thais olet quam non fullonis avari/ testa vetus media sed modo 
ubicadas en las calles como urinarios; también existían los stercorarii, que se encargaban de recoger los residuos ${ }^{44}$ en los plastrum (carros) con que se prestaba el servicio y a los que se alude en la Tabula Heracleensis; dado que uno de los problemas a los que se enfrento Roma era el ruido. Es evidente que existía una contaminación acústica debido al estrépito de la propia ciudad ${ }^{45}$, de ahí que se limitara la circulación por las vías, con algunas excepciones. Veamos la regulación de la Tabula Heracleensis que nos deja entrever lo que desde mi punto de vista constituye un primer conato de normas dirigidas no solo a prevenir el ruido sino también la circulación de vehículos ${ }^{46}$ destinados a la recogida de residuos sólidos, que supone un antecedente en el control de la contaminación acústica; veamos el fragmento:

Tab. Her. 1.56-61: Quae viae in u(bem) R(oman) sunt erunt itra ea loca, ubi continenti bab $<i>t a b<i>t u r$, ne quis in ieis uieis post $k$ (alendas) Ianuar(ias) primas plostrum inter $<r>d i u$ post solem ortum nue ante horam $X$ diei ducito agito, nisi quod aedium sacrarum deorum inmortalium causa aedificandaru $<m>$ operisue publice faciumdei causa adu $<e>$ bei portari oportebit, aut quod ex urbe exue ines loceis earum rerum, quae publice demolienda $<e>$ loca $<$ tae $>$ erunt, publice exportarei oportebit. et quarum rerum causa plostra b(ac)l(ege) certeis bominibus certeis de causeis agere ducere licebit ${ }^{47}$.

La ley establece una serie de excepciones desde el punto de vista de la circulación en carros: "nadie en esas vías después de las calendas de enero podía conducir o llevar carro después del orto o la salida del sol, ni antes de las diez del día", salvo las siguientes excepciones:

- carros destinados a portar material de construcción de obras públicas o para retirar escombros de demoliciones oficiales y construcción de templos.

fracata via. Juvenal III. 34-38: quondam bi cornicines et municipalis barenae perpetui comites notaeque per oppida buccae munera nunc edunt et, verso pollice vulgus cum iubet, occidunt populariter, inde reversi conducunt foricas, et cur non omnia?

44 UlPIANO, 18 ad Sab., D. 7.1.15.1: stercorandis latrinis praeponat.

45 A este problema alude Juvenal con su ingenio satírico respecto de la sociedad romana en III. 232-238: nam quae meritoria somnum/admittunt? magnis opibus ormitur in urbe/ inde caput morbi. raedarum transitus arto/vicorum in flexu et stantis convicia mandrake/eripient somnum Druso vitulisque marinis

46 Olivia F. Robinson. Ancient Rome, cit., p. 81.

47 PaOla PISANI. Uomo, natura, cit., p. 52. Al margen de esta regulación hubo una preocupación por el tráfico peatonal, por ello se procedió a reforzar las vías peatonales para poder descongestionar otras, al mismo tiempo que se potenció la construcción de pórticos, como nos comenta Suetonio Ner. 16: additis porticibus quae frontem insularum protegerent, si bien esto último fue debido a las políticas de remodelación urbanística. En la propia Tab. Her. 1. 68 se menciona cómo deben de estar los pórticos libres para el tráfico peatonal, de manera que estén abiertos y no cerrados: legibus procuratio est erit, ne quis in ieis loceis inue ieis porticibus quid inaedificatum inmolitomue babeto, neue ea loca porticumue quam possideto neue eorum quod saeptum clausumue babeto quo minus eis loceis porticibusque populus utatur pateantu. 
- a lo que se añade (1. 62-65 $\left.{ }^{[48]}\right)$ carros destinados a conducir a las vírgenes vestales, rey de los sacrificios públicos y los flamines.

- carros por razón de triunfo y los reclamados para la celebración de juegos públicos.

Desde el punto de vista de lo que a nuestra materia se refiere, es significativa la 1. 66-67 que señala: Quae plostra noctu in urben inducta erunt, quo minus ea plostra inania aut stercoris, exporandei causa post solem ortum b(oram) X diei bubus iumenteisue iuncta in $u$ (rbe) $R$ (oma) et ab u(rbe) $R$ (oma) p (assus) Messe liceat, eius b (ac)l(ege) mibilum rogatur.

La disposición va referida a los carros que fueran introducidos de noche, para retirar estiércol y otros residuos ${ }^{49}$; no cabe duda de que esta medida supone un control del tráfico rodado ${ }^{50}$ al impedir la circulación de este tipo de vehículos en una franja horaria comprendida entre el orto y las diez, ortum $b$ (oram) X diei, en Roma o a mil pasos de la urbe.

Estos $\operatorname{carros}^{51}$ podían ser también privados, normalmente los procedentes de explotaciones agrícolas, si bien también podían ser de la propia Administración que tenía que contar con un servicio destinado a la purgatio de las calles $^{52}$. Además es importante observar, en relación al transporte de estiércol y otras sustancias, que fue frecuente, como ya hemos apostillado, el reciclaje de algunas de estas sustancias, si bien otras eran evacuadas a través de la red de alcantarillado (foramina).

Quibus diebus virginis Vestales re $<$ gem $>$ sacrorum flamines plostreis in urbe sacrorum publicorum $p$ (opuli) $R$ (omani) caussa uebi oportebit, quaeque plostra triumpbi causa, quo die quisque triumpba $<b>i t$, ducei oportebit, quaeque plostra ludorum <caussa $>$ quei <urbei> romae <p(ropius)ue> urbi Romae propius $<p$ (assus) M> publice, feient, inue pompam ludeis circiensibus ducei agei oput erit, quo[ue] minus earum rerum causa eisque diebus plostra interiu in urbe ducantur agantur, e(ius) b(ac)l(ege) n(ibilium) r(ogantur) Existen varias referencias literarias a este tipo de vehículos, como se desprende, entre otras, de Val. Max. 1.7: "... corpus enim suum a caupone trucidatum tum maxime plaustro ferri ad portam stercore coopertum. tam constantibus familiaris precibus conpulsus protinus ad portam cucurrit et plaustrum, quod in quiete demonstratum erat, conprebendit cauponemque ad capitale supplicium perduxit", y de Tácito, Ann. 11.32: ". . spatium urbis pedibus emensa, vebiculo, quo purgamenta bortorum eripiuntur...".

50 WaRnER ECK. "Verkehr und Verkehrsregeln in einer antiken Grosstadt. Das Beispiel Rom. Stadtverkehr in der antiken Welt", Palilla 18, 2008, p. 61.

51 Existen referencias específicas a los carros que son destinados a la limpieza y recogida de estiércol: UlpiANO, 20 ad Sab., D. 33.7.12.10: "et plaustra quibus stercus evebatur..."; también TÁcıTO, Ann. 11.32.3: "... vebiculo quo purgamenta bortorum excipiuntur...". Sin lugar a dudas, fue el medio de locomoción de transporte urbano por excelencia y también con los que se solían producir numerosos incidentes, como el célebre caso que nos comenta Alfeno del Capitolio, 2 dig., D. 9.2.52.2, en sede ad legem Aquiliam.

52 Se estableció toda una red de alcantarillado para evacuación de residuos procedentes de los baños públicos y las foricae o latrinas, unas 144 aproximadamente, según el Curiosum sobre los regionarios; vid. BARRY HOBSON. Latrinae et foricae. Toilets in the Roman World, London, 2009, p. 5; la evacuación tenía lugar aprovechando la red pública de aguas, para que los

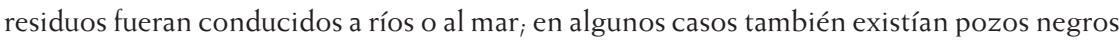
que probablemente eran vaciados por los stercorarii, valiéndose para ello de los carros que hemos señalado. Asimismo, contaban con una red de aguas de reserva que era empleada para la limpieza de las cloacas; vid. FronTinO, Aqued. 2.3. 
En relación con las heces humanas, sabemos que eran recicladas destinándolas a fertilizante para los cultivos ${ }^{53}$, y que su recogida, como hemos visto, obedecía también a una restricción del tráfico viario urbano, con una doble finalidad: por un lado la salubridad ${ }^{54}$ de las personas y por otro el ruido o contaminación acústica ${ }^{55}$.

Además, existía el reciclaje de otro de los residuos orgánicos, la orina humana, dado su poder desengrasante. Fue empleada, sobre todo, en las fulloni$\mathrm{cae}^{56}$, instalaciones dedicadas a la limpieza de tejidos y también de lanas. Por ello fue importante la instalación de recipientes en los que poder recoger la orina para poder luego utilizarla como detergente $y$, de esta forma, eliminar las impurezas de las vestimentas y otros tejidos. El amoniaco de la orina actuaba como desinfectante, al cual se le añadía ceniza que blanqueaba el tejido ${ }^{57}$. Para la gestión de este residuo orgánico, los fullones colocaban ánforas cortadas por la mitad (dolia curta) en la vía pública donde los transeúntes dejaban su contribución. Con la entrada en vigor del nuevo tributo, en el año 70 d. C., esta práctica se abandonó ya que Vespasiano ${ }^{58}$ construyó letrinas públicas donde los propietarios de las fullonicas podían recoger la orina previo pago a los foricari.

53 Vid. VARRO, rust. 1.38.2: Quae loca in agro stercoranda, videndum, et qui et quo genere potissimum facias: nam discrimina eius aliquot. Stercus optimum scribit esse Cassius volucrium praeter palustrium ac nantium. De bisce praestare columbinum, quod sit calidissimum ac fermentare possit terram. Por su parte, Columela, en su obra de re rustica, del año 42 , hace referencia al uso del estiércol humano: $10.85 \ldots$ solido vel stercore aselli/ armentive fimo saturet ieiunia terrae/ ipse ferens bolitor diruptos pondere qualos/pabula nec pudeat fisso praebere novali/ immundis quaecumque vomit latrina cloacis; 11.3.12: Optimum vero stercus est ad bunc usum asini, quia minimum herbarum creat, proximum vel armenti vel ovium, vid. también, entre otras, PlinIO, Nat. Hist. 18.6, y VARRO, rust. 1.6.24; 2.14.1-3,8,5,10.

54 Olivia F. Robinson. Ancient Rome, cit., p. 74

55 Paola Pisani. Uomo, natura, cit., p. 52. Sobre el ruido de la ciudad y el estruendo del hábitat hay numerosas fuentes que dan testimonio de lo estrepitosa que era Roma; vid. MARCIAL $12.57,9.68,10.74,12.57,12.68$. Asimismo, sobre la falta de seguridad por arrojar los desperdicios y residuos desde las ventanas, vid. Juvenal 2.238-248; 3.268-277.

56 Existía toda una compleja red industrial de lavanderías en la periferia de Roma, como nos informa MARCIAL, Ep. 6.93: Tam male Thais olet quam non fullonis auari/testa uetus media, sed modo fracta uia/non ab amore recens bircus, non ora leonis/non detracta cani transtiberina cutis/pullus abortiuo nec cum putrescit in ono/ amphora corrupto nec uitiata garo/Virus ut boc alio fallax permutet odore/deposita quotiens balnea ueste petit/psilothro uiret aut acida latet oblita creta aut tegitur pingui terque quaterque faba/ Cum bene se tutam per fraudes mille putauit/ omnia cum fecit, Thaida Thais olet.

57 PLINIO, Hist. Nat: Surdis etiam rerum sua cuique sunt venena ac minimis quoque pbilyra coci et polline nimium salem cibis eximunt, praedulcium fastidium sal temperat, nitrosae aut amarae aquae polenta addita mitigantur, ut intra duas boras bibi possint, qua de causa in saccos vinarios additur polenta...

58 SUETONIO VeSP. 23.3: Reprehendenti filio Tito, quod etiam urinae vectigal commentus esset, pecuniam ex prima pensione admovit ad nares, sciscitans num odore offenderetur; et illo negante: Atqui, inquit, e lotio est. Nuntiantis legatos decretam ei publice non mediocris summae statuam colosseam, iussit vel continuo ponere, cavam manum ostentans et paratam basim dicens. Ac ne in metu quidem ac periculo mortis extremo abstinuit iocis. Nam cum inter cetera prodigia Mausoleum derepente patuisset et stella crinita in caelo apparuisset, alterum ad Inniam Calvinam e gente Augusti pertinere dicebat, alterum ad Parthorum regem qui capillatus esset, prima quoque morbi accessione: Vae, inquit, puto, deus fio. Paulo en sus resp. 14, D. 22.1.17.5,

Revista digital de Derecho Administrativo, n. ${ }^{\circ} 17$, Primer semestre/2017, PP. 69-87 
Asimismo, encontramos otra serie de problemas en relación con los residuos que no eran orgánicos ni biodegradables, como son los sólidos procedentes del consumo humano, como las ánforas y otros recipientes cerámicos o de vidrio, y también los escombros de obras y edificios. Muchos de ellos eran transportados en barcas cuando se descendía a las marismas de Ostia, ruderi accipiendo Ostienses paludes destinabat, utique naves, quae frumentum Tiberi subvecta[v]issent, onustae rudere decurreren ${ }^{59}$; no es mera coincidencia que otros fueran arrojados directamente al Tíber convirtiendo a este en basuero y provocando cambios en el cauce, lo que hizo que el emperador Augusto interviniese ordenando la limpieza del río que muchas veces sufría desbordamientos a consecuencia de la obstrucción del mismo, como nos informa Suetonio en sus biografías, Aug. 30: Adversus incendia excubias nocturnas vigilesque commentus est, ad coercendas inundationes alveum Tiberis laxavit ac repurgavit completum olim ruderibus et aedificiorum prolationibus coartatum. Como efecto de todo ello fue necesaria la creación específica dentro de la Administración de los curatores alvei et riparum Tiberis, encargados de velar por la limpieza del cauce.

Si tomamos como referencia lo que ya hemos expuesto, lo cierto es que los residuos sólidos procedentes de materiales constructivos y cerámicos terminaban muchas veces en los ríos, en los puertos y en el mar o en vertederos, como el del monte Testaccio. Por ello, es evidente que se acumulaban diferentes tipos de residuos en función de la procedencia: doméstico, industrial derivado de industrias artesanales o de limpieza, como hemos visto en relación a las fullonicas, y el derivado de las obras de construcción, remodelación o derribo de edificios en mal estado, fruto de la actividad edilicia. Excedería de nuestro cometido hacer un análisis pormenorizado de todos ellos, dado que aquí pretendemos sólo hacer una reflexión sobre la gestión y transporte de los mismos.

Como hemos señalado, fue frecuente el uso de los ríos como vertederos y también de zonas próximas a los mismos, pero de igual modo en los puertos encontramos vertidos procedentes de accidentes marítimos, de incidentes de estiba y desestiba de mercancía, e incluso procedentes de la ciudad de Roma que eran transportados en barca, como nos informa Tácito, Ann. 15.43.3: eas proticus Nero sua pecunia exstructurum purgatasque areas dominis traditurum pollicitus est. addidit praemia pro cuiusque ordine et rei familiaris copiis, finivitdue tempus, intra quod effectis domibus aut insulis apiscerentur. ruderi accipiendo Ostienses paludes destinabat, utique naves, quae frumentum Tiberi subvecta[v]issent, onustae rudere decurreren.

O sea, encontramos todo tipo de residuos también en los puertos, no sólo aquellos derivados de la intensa actividad mercantil sino del consumo de las ciudades y de las actividades industriales próximas a los mismos; de ahí la uti-

nos habla de cómo el fisco cobra a los foricarii: Fiscus ex suis contractibus usuras non dat, sed ipse accipit ut solet a foricariis, qui tardius pecunian inferunt... 
lización de barcas especiales que se encargaban de dragar el puerto, mediante ruedas de madera a las que se acoplaban una especie de palas para limpiar el fondo del puerto ${ }^{60}$. Con respecto a la limpieza de puertos y ríos tampoco podemos soslayar la importancia que tuvieron los urinatores ${ }^{61}$, que intervinieron en actividades subacuáticas de diversa índole, al margen de la limpieza, sobre todo en el ámbito de los incidentes marítimos y fluviales.

A mayor abundamiento, los residuos, ya sea orgánicos biodegradables o sólidos, tuvieron también reciclado; ya hemos señalado la importancia de la orina, el estiércol y otros residuos urbanos; si bien el material sólido típico de los vertederos romanos eran las ánforas, junto a escombros procedentes de demoliciones de edificios o insulae. Muchas de esas ánforas fueron reutilizadas para un uso similar como contenedores de almacenaje de líquido y áridos. Sin embargo, otras fueron recicladas para un uso diferente, normalmente para canalización o atarjeas de conducción, pavimentación y aislamiento, como ya hemos comentado con anterioridad, aligeramiento de bóvedas, sellado de muros e impermeabilizaciones, siendo por tanto un material de construcción importantísimo. Es probable que los carros que realizaban la recogida de estiércol ${ }^{62}$, que en ocasiones eran los responsables de la contaminación acústica de la ciudad, fueran los mismos que recogían el material de construcción, es decir, podía existir una recogida selectiva de residuos en función del destino y uso al que podía ir dirigido, si bien creemos que algunas veces no se llegaba a reciclarlos y terminaban siendo arrojados al río o a los puertos, o incluso intramuros ${ }^{63}$.

60 Piero Gianfrotta. "I rifiuti sommersi", Sordes urbis, Roma, 2000, pp. 25 ss.; el autor, hablando de los sordes navales, trae (p. 31, fig. 4) los restos de una embarcación cuya abertura central demuestra la existencia de barcos destinados a dragar el fondo de los puertos. En relación con los restos arqueológicos de este tipo de embarcaciones, vid. estudio cit. por el autor en Patrice POMEY, "Les épaves grecques et romaines de la place Jules-Verne à Marseille", CRAI, 1995, pp. 459-484; en pp. 468-469 alude a ese tipo de embarcaciones cuando señala: "L'existence de ces navires dotés d'un puits central, ouvert sur la mer et surmonté d'un caisson intérieur, était jusqu'à présent totalement inconnue dans l'Antiquité. La question se pose donc de savoir qu'elle pouvait être la fonction de ce puits et l'usage de ces navires. Selon l'ensemble de leurs autres caractéristiques (fond plat, forte membrure, étanchéité importante, nombreuses réparations), il est vraisemblable que l'on est en présence de navires de travail et, d'après le contexte, de bateaux de servitude portuaire".

61 ANDRÉ TCHERNIA, "Les urinatores sur l'épave de la madrague de giens", en Navires et commerces de la Mediterranée Antique, Hommage à Jean Rougé, Lyon: Comité historique du Centre Est, 1988 , pp. 490-497, destaca la recuperación de ánforas. También en extracciones de pecuniae desde finales de la República, Tit. Liv. XLIV.10.3 "Incautior Nicias Pellae proiciendo pecuniae partem quae fuerat ad Phacum sed in re emedabili visus lapsus esse, quod per urinatores omnis ferme extracta est. Tantusque pudor regi pavoris eius fuit ut urinatores clam interfici iusserit...".

62 Quae plostra noctu in urben inducta erunt, quo minus ea plostra inania aut stercoris, exporandei causa post solem ortum b(oram) X diei bubus iumenteisue iuncta in u(rbe) $R$ (oma) et ab u(rbe) $R$ (oma) p(assus) Messe liceat, eius b(ac) l(ege) mibilum rogatur Tab. Her. 66-67.

63 Dentro de la propia ciudad también podían existir vertederos; de hecho, para Antioquía encontramos que en la Oratio 50 de Libanio se alude a la petición al emperador Teodosio 


\section{CONSIDERACIONES FINALES}

Sobre la base de lo anteriormente expuesto, podemos señalar que desde época remota existe una preocupación por la salubridad y el hábitat de las urbes en el derecho romano, con cierta similitud con lo que sucede en nuestra legislación moderna ya que existe, como hemos podido comprobar, una serie de medidas dirigidas a regular la gestión de los vertederos y residuos con el fin de mitigar los efectos perniciosos que estos generan: el amontonamiento de residuos, como vimos con el SC de Pago Montano, una actividad edilicia importante para evitar que se arrojaran residuos a las vías, como se observó en un texto de Papiniano, D. 43.10.1.5, o en muchos de los pasajes de la Tabulae Heracleensis, donde se habla del mantenimiento, limpieza o purgatio e incluso de refectio. Sin olvidar los diferentes interdictos creados por el pretor ex profeso para la tutela de la salubridad.

Además, hemos podido verificar la existencia de todo un servicio de recogida de residuos, por parte de los extractores oforicarii, junto a los stercorarii que se encargaban de gestionar los residuos, con un control al que se refiere la Tab. Her. 1. 56-61, que fija un horario de transporte o paso de los carros o plastrum con la finalidad de evitar no solo la acumulación de residuos sino, nos atreveríamos a decir, evitar el ruido en las ciudades, es decir, la contaminación acústica.

Finalmente, no podemos soslayar en esta breve aproximación la existencia de cierta cultura de reciclaje para determinado tipo de residuos líquidos, orgánicos y sólidos, en este último caso sobre todo de ánforas y vidrio, normalmente relacionada con la intensa actividad mercantil y de transporte, lo que probablemente derivó en la adopción de normas que evitaran la acumulación de restos de construcción y material cerámico en las ciudades, y que muchas veces también pudo derivar en recogida selectiva de residuos, como sucede en la actualidad.

\section{BIBLIOGRAFÍA}

Albertario, Emilio. "L'uso traslato di salubris, salubritas, salubriter nelle fonti giuridiche romane", RFIC, 57, 1929, pp. 86-96.

Bisphant, EDward. "The End of the Tabula Heracleensis a por man's sanctio?", Epigrapbica, 59, 1997.

Casavola, Franco. Studi sulle azione popolari romani. Les actiones populares, Napoli, 1958.

Coarelli, Filippo. Roma, Roma-Bari: Laterza, 1981.

para que obligue a los campesinos que llegan con sus carros a llevarse restos de construcción y residuos fuera de la ciudad. 
Crawford, Michael H. Roman Statutes, London, vol. I, 1996.

D'Ors, Álvaro. "Nuevos datos de la ley Irnitana sobre jurisdicción municipal", SDHI $49,1983$.

De Magistris, Elio. Structurae, ricerche su tecniche costruttive e monumento antichi, Napoli, 2009.

Dressel, HeInRICH. Inscriptiones Urbis Romanae Latinae-Instrumentum domesticum 1-2, Berlin, 1966-1975.

ECK, Warner. Die Verwaltung des Römischen Reiches in der bohen Kaiserzeit, I, Basel, 1995.

ECK, WARnER. "Verkehr und Verkehrsregeln in einer antiken Grosstadt. Das Beispiel Rom. Stadtverkehr in der antiken Welt", Palilla 18, 2008, 61.

Fernández de Buján, Antonio. Derecho público romano, Madrid, 2016.

Fernández de BujÁn, Antonio. "Instituciones, hechos y actividad de orden administrativo en la experiencia jurídica romana", Xornadas e Seminarios: Derecho Administrativo Histórico, 1, 2005.

Fischer, Renate. Umweltschützende Bestimmungen im Römischen Recht, Aachen: Shaker, 1996.

Gianfrotta, Piero. "I rifiuti sommersi", Sordes urbis, Roma, 2000.

Hobson, BARry. Latrinae et foricae. Toilets in the Roman World, London, 2009.

Impallomeni, Giambattista. "L'inquadramento giuridico delle colonie e dei municipi (Iula Concordia e Opitergium) nell'ambito dell'Impero Romano", Index 26, 1998.

Mangas, Julio. Leyes coloniales y municipales de la Hispania romana, Madrid, 2001.

Palma, Antonio. Iura vicinitatis, Solidarietà e limitazioni nel rapporto di vicinato in diritto romano dell'età classica, Torino, 1988.

Panciera, Silvio. "Nettezza urbana a Roma. Organizzazione e responsabile", Sordes Urbis, Roma, 2000.

PisAnI, PAOla. Uomo, natura, ambiente nella letteratura latina, Genova: Compagnia dei librai, 1990.

Pomey, PAtrice. "Les épaves grecques et romaines de la place Jules-Verne à Marseille", CRAI, 1995, p. 459.

Priester, Sascha. Ad summas tegulas. Untersucbungen zu vielgeschossigen Gebäudeblöcken mit Wobneinbeiten und Insulae im Kaiserzeitlichen Rom, Roma, 2002. 
Pugliese, Giovanni. "Figure processuali ai confini tra iudicia privata e iudicia publica", Studi Solazzi, Napoli, 1948, p. 411.

Robinson, Olivia F. Ancient Rome. City planning and administration, London, 1992.

Rodríguez González, Ana. "Reception of Law: derecho y espacio urbano en dos ciudades de la Antigüedad", Rida, vol. 59, 2012, pp. 23 ss.

Scobie, Alexander. "Slums, sanitation and morality in the roman world", Klio, vol. $68,{ }^{\circ}{ }^{2} 2,1986$, pp. 412 ss.

Solidoro Maruotti, LaURA. La tutela dell'ambiente nella sua evoluzione storica, L'esperienza del mondo antico, Torino, 2009.

TCHERnia, ANDrÉ. "Les urinatores sur l'épave de la madrague de giens", en Navires et commerces de la Méditerranée antique, Hommage à Jean Rougé, Lyon: Comité historique du Centre Est, 1988, pp. 490-497. 